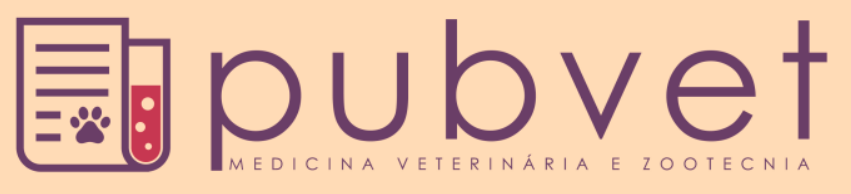

https://doi.org/10.31533/pubvet.v12n9a178.1-7

\title{
Nefrectomia unilateral em um cão parasitado por Dioctophyma renale: Relato de caso
}

\author{
Dilma Mendes de Freitas $^{1 *}$, Bruna Piva Maria ${ }^{2}$, Bárbara Michelle Araújo \\ Vasconcelos $^{30}$, Ana Luiza Teixeira Amado Jorge ${ }^{40}$, Ananda Neves Teodoro ${ }^{50}$, \\ Endrigo Gabellini Leonel Alves ${ }^{6}{ }^{\bullet}$, Isabel Rodrigues Rosado ${ }^{7}$ \\ ${ }^{I}$ Médica Veterinária. Uberaba - MG Brasil. *Autor para correspondência. E-mail: dilmamendes3m@yahoo.com.br \\ ${ }^{2}$ Médica Veterinária, Hospital Veterinário de Uberaba - Setor Pequenos Animais. Uberaba - MG Brasil. E-mail:brupiva@hotmail.com \\ ${ }^{3}$ Residente do Programa de Aprimoramento em Clínica Cirúrgica de Pequenos Animais do Hospital Veterinário de Uberaba. Uberaba - MG \\ Brasil. E-mail: barbaravasconcellos4@gmail.com \\ ${ }^{4}$ Residente do Programa de Aprimoramento em Clínica Cirúrgica de Pequenos Animais do Hospital Veterinário de Uberaba. Uberaba-MG \\ Brasil.E-mail:analuiza_jorge@hotmail.com \\ ${ }^{5}$ Residente do Programa de Aprimoramento em Anestesiologia Veterinária do Hospital Veterinário de Uberaba. Uberaba - MG Brasil. E- \\ mail: ananda.tteodoro@gmail.com \\ ${ }^{6}$ Coordenador do Mestrado em Sanidade de produção animal nos trópicos da Universidade de Uberaba. Uberaba - MG Brasil. E- \\ mail:endrigoglalves@gmail.com \\ ${ }^{7}$ Docente do Mestrado em Sanidade de produção animal nos trópicos da Universidade de Uberaba. Uberaba - MG Brasil. E- \\ mail:isabel.rosado@uniube.br
}

\begin{abstract}
RESUMO. O Dioctophyma renale é um helminto que parasita os rins dos cães e cuja a infecção é adquirida pela ingestão de larvas que podem estar presentes em peixes, rãs ou anelídeos aquáticos. O rim direito e a cavidade abdominal são os locais onde o parasita é mais encontrado. Os sinais clínicos em geral são hematúria, inapetência e dores lombares, porém, os animais podem ser assintomáticos quando apenas um rim é parasitado. $\mathrm{O}$ diagnóstico é realizado através da ultrassonografia, urinálise e urografia excretora e o tratamento consiste na nefrectomia para casos em estágio avançado ou nefrotomia para retirada do parasita em casos com diagnóstico precoce. O presente trabalho descreve o caso de uma cadela, sem raça definida, com 2 anos de idade, proveniente de fazenda, atendida no Hospital Veterinário de Uberaba e diagnosticada com o Dioctophyma renale por meio de ultrassonografia e urinálise. O tratamento foi realizado por nefrectomia unilateral com excelente recuperação pós-operatória.
\end{abstract}

Palavras-chave: nematoide, rins, ultrassonografia, urinálise

\section{Unilateral nephrectomy in a dog parasitized by Dioctophyma renale: Case report}

\begin{abstract}
Dioctophyma renale is a helminth that parasites the kidneys of dogs and whose infection is acquired by ingestion of larvae that may be present in fish, frogs or aqua annelids. The right kidney and abdominal cavity are the places where the parasite is most commonly found. The clinical signs in general are hematuria, inappetence and low back pain, however, the animals can be asymptomatic when only one kidney is parasitized. The diagnosis is made through ultrasonography, urinalysis and excretory urography and the treatment consists of nephrectomy for advanced cases or nephrectomy for removal of the parasite in cases with early diagnosis. The present study describes the case of a 2 year old bitch from a farm attended at the Veterinary Hospital of Uberaba and diagnosed with Dioctophyma renale by ultrasound and urinalysis. The treatment was done by unilateral nephrectomy with excelent postoperative recovery.
\end{abstract}

Keywords: nematoid, kidneys, ultrasonography, urinalysis 


\section{Nefrectomía unilateral en un perro parasitado por Dioctophyma renale: Relato de caso}

RESUMEN. El Dioctophyma renale es un helminto que paraliza los riñones de los perros y cuya infección se adquiere por la ingestión de larvas que pueden estar presentes en peces, ranas o anélidos acuáticos. El riñón derecho y la cavidad abdominal son los lugares donde el parásito es más encontrado. Los signos clínicos en general son hematuria, inapetencia y dolores lumbares, sin embargo, los animales pueden ser asintomáticos cuando sólo un riñón es parasitado. El diagnóstico se realiza a través de la ultrasonografía, uroanálisis y urografía excretora y el tratamiento consiste en la nefrectomía para casos en etapa avanzada o nefrotomía para retirada del parásito en casos con diagnóstico precoz. El presente trabajo describe el caso de una perra, sin raza definida, con 2 años de edad, proveniente de hacienda, atendida en el Hospital Veterinario de Uberaba y diagnosticada con el Dioctophyma renale por medio de ultrasonografía y uroanálisis. El tratamiento fue realizado por nefrectomía unilateral con excelente recuperación postoperatoria.

Palabras clave: nematodo, riñones, ultrasonografía, uroanálisis

\section{Introdução}

O Dioctophyma renale é um nematoide conhecido como o maior verme do rim, podendo medir até um metro de comprimento (Alves et al., 2007; Sousa et al., 2011; Chauhan et al., 2016). Este nematoide pode parasitar mamíferos domésticos, silvestres e seres humanos (Kommers et al., 1999; Pedrassani \& Nascimento, 2015).

O hospedeiro definitivo se infeta ao ingerir anelídeos, sapos, peixes ou rãs ou peixes parasitados com a forma larval (Varzone et al., 2008). Nesses hospedeiros, os vermes adultos se reproduzem nos rins e eliminam os ovos na urina (Kommers et al., 1999, Pedrassani \& Nascimento, 2015). Uma vez no ambiente, a primeira forma larval se desenvolve no interior dos ovos que são ingeridos pelo hospedeiro intermediário, um anelídeo oligoqueta aquático (Lumbriculus variegatus) (Kommers et al., 1999; Nakagawa et al., 2007; Verocai et al., 2009; Pedrassani \& Nascimento, 2015). No anelídeo a larva sofre alterações morfológicas atingido a fase L3. O hospedeiro paratênico (peixes ou sapos) se infectam ao ingerir esse anelídeo, e o ciclo se completa quando o cão ou outro hospedeiro definitivo ingere o hospedeiro paratênico (Karmanova, 1968).

No hospedeiro definitivo, além dos rins, os parasitas podem estar em outros locais da cavidade abdominal e torácica (Kommers et al., 1999; Monteiro et al., 2002; Zabott et al., 2012; Pedrassani \& Nascimento, 2015). O rim direito é acometido com maior frequencia devido a sua proximidade com o duodeno (Kommers et al., 1999; Anderson, 2000).
Os sinais clínicos mais frequentes são hematúria, disúria, depressão e dor lombar (Pedrassani \& Nascimento, 2015; Silveira et al., 2015).

O diagnóstico é feito por meio de observação dos ovos do parasita na urinálise e pela visibilização do nematódeo por ultrassonografia (Zardo et al., 2012; Pedrassani \& Nascimento, 2015).

O tratamento definitivo é realizado por nefrotomia para retirada do parasita nos casos diagnosticados precocemente ou por nefrectomia em casos tardios em que o parênquima renal está completamente destruído (Alves et al., 2007; Kommers et al., 1999; Sousa et al., 2011).

A prevenção dessa enfermidade se dá por meio de medidas como evitar a ingestão de peixes e rãs maus cozidos, sapos, anelídeos ou água de rios (Measures, 2001; Varzone et al., 2008).

O objetivo deste trabalho é relatar um caso de uma cadela com dois anos de idade parasitada por Dioctophyma renale, abordando as manifestações clínicas, o diagnóstico e tratamento.

\section{Relato de caso}

Foi atendida no Hospital Veterinário de Uberaba, uma cadela, sem raça definida, de dois anos de idade, com massa corporal de $36,8 \mathrm{~kg}$. O tutor levou o animal para realização de ovariohisterectomia eletiva pois acreditava que ela estava no cio devido a secreção vulvar sanguinolenta. A cadela não possuía histórico de doenças, não havia sido vacinada, mas a desverminação e o controle de ectoparasitas haviam sido realizados recentemente. $\mathrm{O}$ animal apresentava normorexia, normodipsia, normoquesia e normúria e alimentava-se de ração 
e comida caseira. A cadela era proveniente de uma fazenda do município de Uberaba.

No exame clínico geral observou-se estado mental, comportamento e escore corporal normais. As mucosas estavam normocoradas e úmidas. $\mathrm{O}$ tempo de preenchimento capilar foi de 2 segundos e os linfonodos não apresentavam-se alterados à palpação. A frequência cardíaca era $120 \mathrm{bpm}$, a respiratória $20 \mathrm{mpm}$ e a temperatura retal $39,4^{\circ} \mathrm{C}$. Não foram identificadas alterações á palpação abdominal mas observou-se secreção vulvar sanguinolenta.

Foram realizados hemograma e bioquímico sérica com avaliação de alanina aminotransferase (ALT) e creatinina. No hemograma observou-se leucocitose de $23.100 \mathrm{cel} / \mathrm{mm}^{3}$ (referência - 6.000 A $18.000 \mathrm{~mm}^{3}$ ), linfocitose de $6.468 \mathrm{cel} / \mathrm{mm}^{3}$ (referência - 720 A $5.400 \mathrm{~mm}^{3}$ ) e eosinofilia 5.082 cel $/ \mathrm{mm}^{3}$ - (referência - 120 A $1.800 \mathrm{~mm}^{3}$ ). Na análise bioquímica a ALT e creatinina estavam dentro da normalidade.

Realizou-se ultrassonografia abdominal para investigar a origem da leucocitose. Foram visibilizadas múltiplas imagens tubulares hiperecogênicas com centro anecogênico, compatíveis com Dioctophyma renale, ocupando grande parte do rim direito (Figura 1). O rim esquerdo apresentava parênquima de aspecto normal, relação cortico-medular preservada e dimensões de 7,34 cm no maior eixo longitudinal (Figura 2). O útero estava com dimensões normais sem conteúdo no lúmen (Figura 3 ).

Realizou-se urinálise e a análise do sedimento permitiu a observação de ovos operculados, de cor acastanhada, parede espessada e irregularidade na sua superfície, característicos de Dioctophyma renale (Figura 4). Além disso, a urina apresentava-se com coloração amarelo escuro, aspecto turvo, pH 5,0 (referência - 5,5 A 7,5), proteinúria, sangue oculto, células escamosas e transicionais, leucócitos, hemácias, cilindros granulosos raros e microbiota bacteriana intensa.

$\mathrm{O}$ animal foi encaminhado para laparotomia exploratória e nefrectomia. A medicação préanestésica (MPA) foi realizada com metadona $(0,3 \mathrm{mg} / \mathrm{kg}), \quad$ dexmedetomidina $\quad(2 \mu \mathrm{l} / \mathrm{kg}) \quad \mathrm{e}$ cetamina $(5 \mathrm{mg} / \mathrm{kg})$, por via intramuscular. A indução anestésica foi realizada com propofol $(5 \mathrm{mg} / \mathrm{kg})$ e fentanil $(5 \mu \mathrm{l} / \mathrm{kg})$, via intravenosa, seguida por intubação endotraqueal para manutenção com isofluorano $0,8 \%$. Durante todo $\mathrm{o}$ procedimento o animal recebeu fentanil em infusão contínua variando entre 0,02 a 0,04 $\mu \mathrm{l} / \mathrm{kg} /$ minuto de acordo com a resposta obtida.

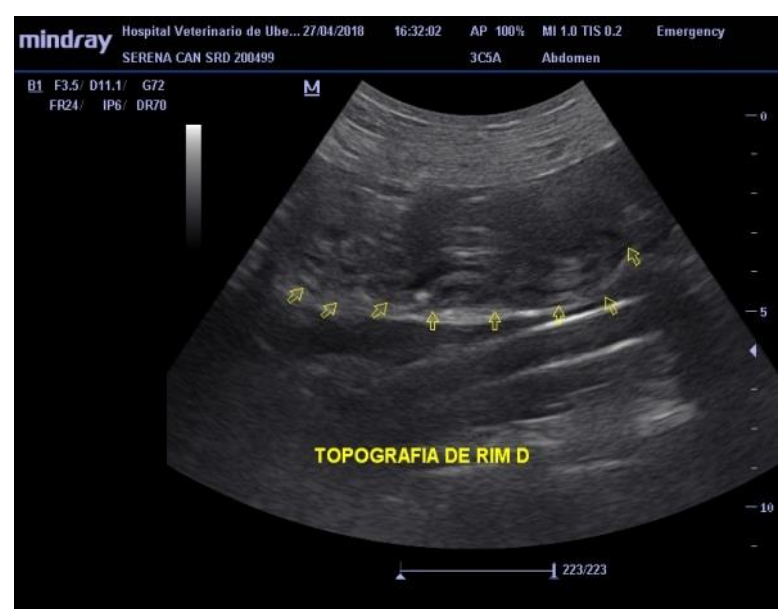

Figura 1. Imagem de ultrassonografia abdominal de uma cadela SRD, de 2 anos de idade, com leucocitose e secreção vulvar sanguinolenta. Nota-se em topografia de rim direito múltiplas imagens tubulares hiperecogênicas com centro anecogênico compatíveis com Dioctophyma renale (setas amarelas).

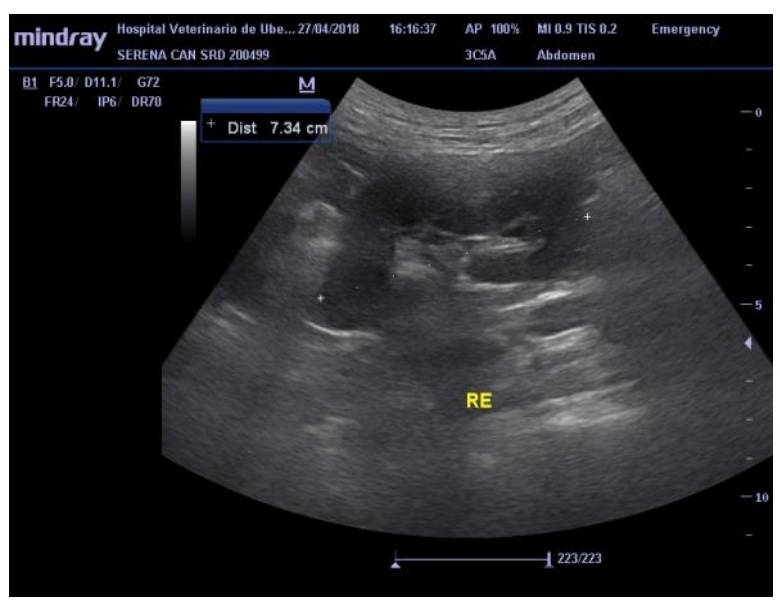

Figura 2. Imagem de ultrassonografia abdominal de uma cadela SRD, de 2 anos de idade, com leucocitose e secreção vulvar sanguinolenta. Nota-se rim esquerdo mensurando 7,34 $\mathrm{cm}$ no maior eixo longitudinal com relação corticomedular preservada e ecogenicidade da cortical mantida.

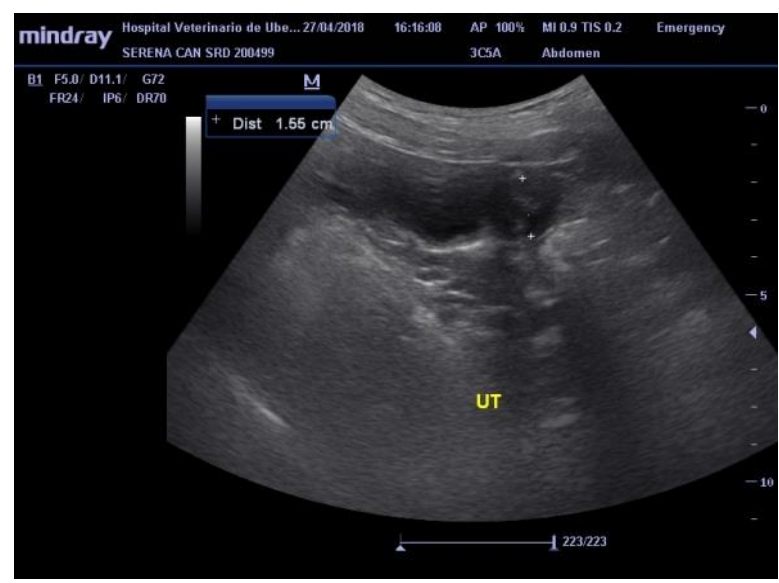

Figura 3. Imagem de ultrassonografia abdominal de uma cadela SRD, de 2 anos de idade, com leucocitose e secreção vulvar sanguinolenta. Nota-se útero com dimensões normais sem conteúdo no lúmen. 


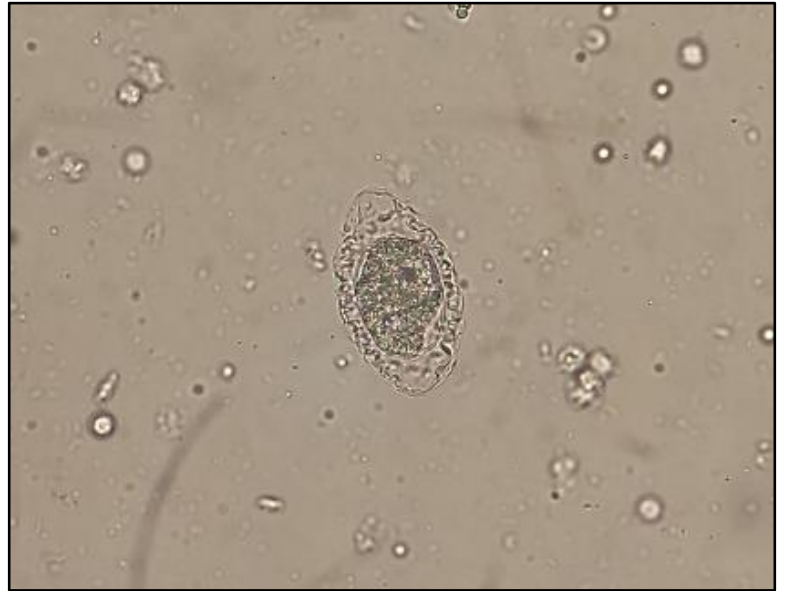

Figura 4. Fotomicroscopia de Ovo de Dioctophyma renale presente em sedimento de urina de uma cadela SRD, de 2 anos de idade, com leucocitose e secreção vulvar sanguinolenta (Aumento 40X). Nota-se ovo operculado, cor acastanhada, parede espessada e irregularidades na sua superfície.

Foi administrado cefazolina (30 $\mathrm{mg} / \mathrm{kg})$ e meloxicam $(0,2 \mathrm{mg} / \mathrm{kg})$, por via intravenosa. Realizou-se a preparação da área cirúrgica asséptica, com ampla tricotomia e antissepsia com clorexidine degermante $2 \%$ e alcoólico $0,5 \%$. Procedeu-se incisão cutânea em região média ventral pré-retro-umbilical, a linha alba foi incisada para acesso á cavidade abdominal. O rim direito foi localizado e apresentava perda da morfologia (Figura 5A).

Haviam aderências em topografia renal, diante disso prosseguiu-se sua dissecação. Realizou-se dupla ligadura da artéria, veias renais e do ureter, com fio inabsorvível sintético monofilamentar (Nylon 0, Ethicon, Brasil), seguida pela secção das estruturas ligadas e excisão do rim (Figura $5 \mathrm{~B}$ e C).

Em seguida realizou-se inspeção minuciosa das vísceras abdominais, uma vez que o nematóide pode ser encontrado também na cavidade peritoneal. A cadela foi submetida a ováriohisterectomia.

Para ráfia da musculatura abdominal foi usado fio absorvível sintético monofilamentar (Caprofyl 0, Ethicon, Brasil) no padrão Sultan; a sutura do subcutâneo foi realizada com padrão ZigueZague, com fio (Caprofyl 2-0, Ethicon, Brasil)e a dermorrafia foi feita com sutura em padrão Wolf, com fio Nylon 3-0 (Ethicon, Brasil).

O rim retirado foi inspecionado e haviam três nematoides Dioctophyma renale adultos. Havia perda completa do parênquima renal, moderada quantidade de liquido sanguinolento e espessamento da cápsula (Figura $5 \mathrm{D}$ e E).

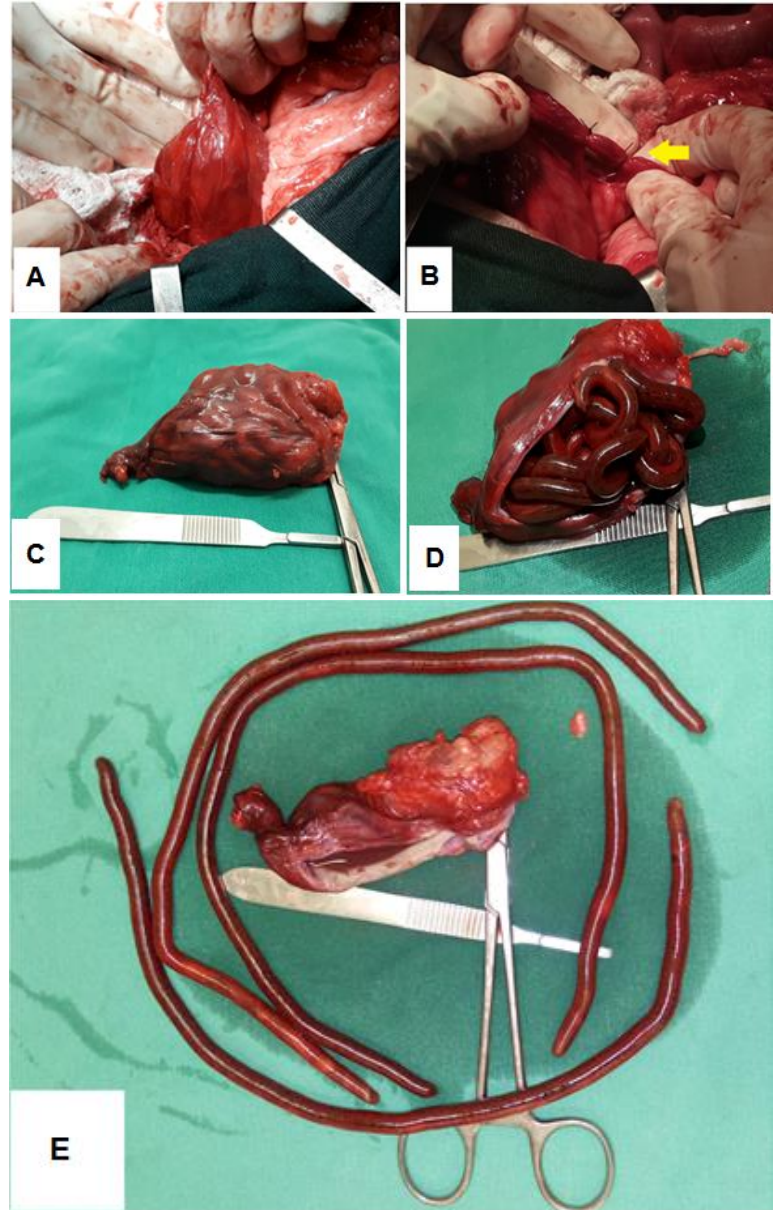

Figura 5. Imagem do procedimento de nefrectomia do rim direito em uma cadela SRD, de 2 anos de idade acometida por Dioctophyma renale. A) Exposição do rim direito mostrando alteração de sua morfologia. B) Ligadura em artéria e veia renal (seta amarela). C) Rim direito após nefrectomia. D) Incisão em cápsula renal e exposição dos helmintos. E) Presença de três parasitas de Dioctophyma renale e líquido sanguinolento no interior do rim.

No pós-operatório o animal ficou internado em observação por três dias. $\mathrm{O}$ tratamento foi realizado com metadona $(0,1 \mathrm{mg} / \mathrm{kg}$, QID, por 2 dias $)$, cloridrato de tramadol $(4 \mathrm{mg} / \mathrm{kg}$, TID, por $1 \mathrm{dia})$, cefazolina $(30 \mathrm{mg} / \mathrm{kg}$, TID, por 3 dias $)$, dipirona sódica $(25 \mathrm{mg} / \mathrm{kg}$, TID, por 3 dias) omeprazol (1 $\mathrm{mg} / \mathrm{kg}$, SID, por 3 dias) e fluidoterapia com ringer com lactato ( $3 \mathrm{~mL} / \mathrm{kg} / \mathrm{hora}$, por 3 dias). Avaliou-se ureia e creatinina, que se encontravam dentro da normalidade.

O animal teve alta com prescrição de omeprazol (1 mg/kg, por VO, SID, por 7 dias), amoxicilina com clavulanato de potássio (20 $\mathrm{mg} / \mathrm{kg}$, por VO, BID, por 7 dias), cloridrato de tramadol (4 mg/kg, por VO, BID, por 5 dias), dipirona ( $25 \mathrm{mg} / \mathrm{kg}$, por VO, BID, por 5 dias) e limpeza da ferida cirúrgica diariamente com solução fisiológica seguida de aplicação de clorexidine em spray. 
O proprietário relatou que o animal apresentou excelente recuperação no pós-operatório.

\section{Discussão}

$\mathrm{O}$ animal do presente relato era proveniente de zona rural, não tinha acesso a rios, mas tinha histórico de viver em uma fazenda onde existiam sapos. Há relatos de que a infecção pode ocorrer por meio da ingestão de rãs, além de peixes ou anelídeos aquáticos contendo a forma larval do parasita (Varzone et al., 2008; Pedrassani \& Nascimento, 2015), portanto a ingestão de sapo pode ter sido a forma de infecção.

O único achado no exame clínico observado na cadela deste relato foi a secreção vulvar sanguinolenta que pode ter sido por cio. Portanto, não foram observadas nenhuma das manifestações clínicas já descritas como hematúria, disúria, hemoperitônio, peritonite e uremia (Gargili et al., 2002; Leite et al., 2005; Newman, 2013). Há relatos de casos em que o nematódeo foi encontrado como um achado durante a necropsia, logo alguns animais podem ser assintomáticos, principalmente quando apenas um dos rins é acometido (Monteiro et al., 2002; Alves et al., 2007). O diagnóstico ante mortem do animal do presente relato permitiu a realização do tratamento efetivo prevenindo a evolução para um quadro de insuficiência renal ou a ocorrência de complicações, como por exemplo, ruptura da cápsula renal.

Os exames bioquímicos deste animal se encontravam dentro dos parâmetros normais, provavelmente porque apenas um dos rins estava acometido. Uma alteração bioquímica que pode ser encontrada em animais com Dioctophyma renale é a azotemia em razão de uma insuficiência renal, caso ambos os rins estejam acometidos ou se apenas um esteja afetado e o outro prejudicado devido a alguma comorbidade (Bichard \& Sherding, 2003; Newman, 2013).

No hemograma do paciente deste estudo foi encontrado leucocitose por eosinofilia que coincide com a descrição de achados hematológicos já relatados por outros autores (Bichard \& Sherding, 2003; Newman, 2013).

O diagnóstico definitivo desse animal foi realizado pela visibilização do nematódeo pela ultrassonografia e dos ovos do parasita na urinálise, assim como descrito por outros autores (Zardo et al., 2012; Pedrassani \& Nascimento, $\underline{2015}$ ). Além disso, a realização da urinálise permitiu a observação de hemácias, leucócitos e proteínas provavelmente devido a lesão e inflamação do rim. Isso mostra que a urinálise é importante não só para o diagnóstico, mas também para definir o prognóstico e a escolha do tratamento (Bichard \& Sherding, 2003; Thrall et al., 2015).

No presente caso clínico não se optou pela realização da urografia excretora devido ao risco de induzir lesão no rim não parasitado. $\mathrm{O}$ meio de contraste iodado usado durante a urografia excretora possui maior viscosidade que o sangue, o que diminui o fluxo sanguíneo, induz a vasoconstrição renal, reduzindo o fluxo urinário que leva a concentração do contraste causando com isso citotoxicidade das células tubulares renais (Wong \& Irwin, 2007; Seeliger et al., 2012).

O tratamento da dioctofimose renal com um anti-helmíntico não é eficaz pois mesmo que o $D$. renale seja morto pela ação do anti-parasitário ele não é eliminado do rim devido ao seu tamanho e espessura (Pedrassani et al., 2009; Zolhavarieh et al., 2016). Por esse motivo deve-se sempre optar pelo tratamento cirúrgico.

No paciente em questão apenas o rim direito se encontrava parasitado e com perda da maior parte do seu parênquima, portanto optou-se pela nefrectomia. Outros relatos de dioctofimose renal descrevem que o tratamento recomendado para casos com diagnóstico precoce é a retirada cirúrgica do nematódeo por nefrotomia. Entretanto a nefrectomia é recomendada quando há hidronefrose e o rim oposto apresenta funcionamento normal (Kommers et al., 1999; Ferreira et al., 2010; Sousa et al., 2011; Gómez, 2017). Em carnívoros, o rim direito é o local mais parasitado pelo $D$. renale, seguido pela cavidade abdominal (Gargili et al., 2002). Esta predileção ocorre porque as larvas localizadas no duodeno migram para rim direito pela sua proximidade anatômica (Kommers et al., 1999; Anderson, 2000; Zabott et al., 2012).

A nefrectomia foi realizada como descrito por Gómez et al. (2017). A gordura peritoneal foi afastada para facilitar a identificação do rim, seguida por divulsão cuidadosa das estruturas do hilo para não lesar veia e artéria renal, o que poderia levar a hemorragia grave e morte do paciente. O ureter também deve ser ligado e seccionado. Quando há hidroureter recomenda-se que essa ligadura seja realizada o mais próximo 
possível da bexiga para minimizar as complicações pós-operatórias como cistite.

A avaliação macroscópica do rim acometido revelou destruição completa do parênquima renal com preservação apenas da cápsula, a qual se apresentava com aspecto irregular e com exsudato sanguinolento. Os mesmos achados macroscópicos também foram observados em outros casos de dioctofimose relatados anteriormente (Kommers et al., 1999; Anderson, 2000; Leite et al., 2005). A destruição do parênquima renal ocorre por ação de enzimas proteolíticas e lipolíticas produzidas por glândulas esofagianas do nematódeo (Nakagawa et al., 2007).

Não foi possível determinar se o sangramento vulvar era proveniente da hematúria ou se o animal estava no cio. Um exame que poderia ter sido realizado é a citologia vaginal, uma vez que esse exame permite determinar em qual fase do ciclo estral o animal se encontra (Holst \& Phemister, 1975).

Conclui-se que a dioctofimose renal pode ser assintomática em alguns casos, devendo ser incluída como diagnóstico diferencial em casos que apresentem leucocitose por eosinofilia. A ultrassonografia, urinálise e bioquímica renal são exames complementares indispensáveis para o diagnóstico e tratamento do paciente com Dioctophyma renale. A nefrectomia é um tratamento eficiente para a dioctofimose unilateral quando $o$ paciente apresenta função renal preservada.

\section{Referências bibliográficas}

Alves, G. C., Silva, D. T. \& Neves, M. F. 2007. Dioctophyma renale: O parasita gigante do rim. Revista Científica Eletrônica de Medicina Veterinária. Garça, 8, 1-5.

Anderson, R. C. 2000. Nematode parasites of vertebrates: their development and transmission (2nd ed.). CABI Publishing, Wallingford, Oxon, UK.

Birchard, S. J. \& Sherding, R. G. 2003. Manual Saunders: Clínica de Pequenos Animais (2a ed.). Roca, São Paulo, BR.

Chauhan, S., Kaval, S. \& Tewari, S. 2016. Dioctophymiasis: A rare case report. Journal of Clinical and Diagnostic Research, 10, 1-2.

Ferreira, V. L., Medeiros, F. P., July, J. R. \& Raso, T. F. 2010. Dioctophyma renale in dog: clinical diagnosis and surgical treatament. Veterinary Parasitology, 168, 151- 155.

Gargili, A., Firat, I., Toparlak, M. \& Çetinkaya H. 2002. First case report of Dioctophyme renale (Goeze, 1782) in a dog in İstanbul, Turkey. Turkish Journal of Veterinary and Animal Sciences, 26, 189-1191.

Gómez, J. R., Sanudo, M. J. M. \& Morales, J. G. 2017. Cirurgia na clínica de pequenos animais, a cirurgia em imagens passo a passo: abdome caudal. MedVet, São Paulo, BR.

Holst, P. A. \& Phemister, R. D. 1975. Temporal sequence of events in the estrous cycle of the bitch. American Journal Veterinary Research, 36, 705-706.

Karmanova, E. M. 1968. Fundamentals of nematology. In: Dioctophymatoidea of animals and man and diseases caused by them. Nauka Publishers, Moscow, Russian.

Kommers, G. D., Ilha, M. R. S. \& Barros, C. S. L. 1999. Dioctofimose em cães: 16 casos. Ciência Rural, 29, 517-522.

Leite, L. C., Círio, S. M., Diniz, J. M. F., Luz, E., Silva, M. A. N., Silva, A. W. C., ... \& Pereira, C. C. 2005. Lesões anatomopatológicas presentes na infecção por Dioctophyma renale (Goeze, 1782) em cães domésticos (Canis familiaris, Linnaeus, 1758). Archives of Veterinary Science, 10, 95-101.

Measures, L. N. 2001. Dioctophymatosis. In: Samuel, W. M., Pybus, M. J. \& Kocan, A. A. (eds), Parasitic diseases of wild mammals (2nd ed). Iowa State University Press, Ames, US.

Monteiro, S. G., Sallis, E. S. V. \& Stainki, D. R. 2002. Infecção natural por trinta e quatro helmintos da espécie Dioctophymarenale (Goeze, 1782) em um cão. Revista da Faculdade de Zootecnia, Veterinária e Agronomia, 9, 95-99.

Nakagawa, T. L. D. R., Bracarense, A. P. F. R. L., Reis, A. C. F., Yamamura, M. H. \& Headley, S. A. 2007. Giant kidney worm (Dioctophyma renale) infections in dogs from Northern Paraná, Brazil. Veterinary Parasitology, 145, 366-370.

Newman, S. J. 2013. O sistema urinário. In: Zachary J. F. \& Mcgavin, M. D. Bases da Patologia Veterinária. Elsevier, São Paulo, Brasil.

Pedrassani, D., Hoppe, E. G. L., Avancini, N. \& Nascimento, A. A. N. 2009. Morphology of eggs of Dioctophyme renale Goeze, 
1782(Nematoda: Dioctophymatidae) and influences of temperature on development of first-stage larvae in the eggs. Revista Brasileira de Parasitologia Veterinária, 18, 15-19.

Pedrassani, D. \& Nascimento, A. A. 2015. Verme gigante renal. Revista Portuguesa de Ciências Veterinárias, 110, 30-37.

Seeliger, E., Sendeski, M., Rihal, C. S. \& Persson, P. B. 2012. Contrast-induced kidney injury: mechanisms, risk factors and prevention. European Heart Journal, 33, 2007-2015.

Silveira, C. S., Diefenbach, A., Mistieri, M. L., Machado, I. R. L. \& Anjos, B. L. 2015. Dioctophyma renale em 28 cães: aspectos clinicopatológicos e ultrassonográficos. Pesquisa Veterinária Brasileira, 35, 899-905.

Sousa, A. A. R., Sousa, A. A. S., Coelho, M. C. O. C., Quessada, A. M., Freitas, M. V. M. \& Moraes, R. F. N. 2011. Dioctofimose em cães. Acta Scientiae Veterinariae, 39, 1-4.

Thrall, M. A., Weiser, G., Allison, R. W. \& Campbell, T. W. 2015. Hematologia $e$ Bioquímica Clínica Veterinária (2a ed.). Guanabara Koogan, Rio de Janeiro, BR.

Varzone, J. R. M., Aquino, L. P. C. T. \& Rodovalho, M. V. T. 2008. Achados macroscópicos de lesões resultantes do parasitismo por Dioctophyma renale em loboguará (Chrysocyon brachyurus) - Relato de Caso. Ensaio e Ciência: Ciências Biológicas, Agrárias e da Saúde, 12, 171-178.
Verocai, G. G., Measures, L. N., Azevedo, F. D., Correia, T. R., Fernandes, J. I. \& Scotty, F. B.. 2009. Dioctophyma renale (Goeze, 1782) in the abdominal cavity of a domestic cat from Brazil. Veterinary Parasitology, 161, 342-344.

Wong, G. T. C. \& Irwin, M. G. 2007. Contrastinduced nephropathy. British Journal of Anaesthesia, 99, 474- 483.

Zabott, M. V., Pinto, S. B., Viott, A. M., Tostes, R. A., Bittencourt, L. H. F. B., Konell, A. L. \& Gruchouskei, L. 2012. Ocorrência de Dioctophyma renale em Galictis cuja. Pesquisa Veterinária Brasileira, 32, 786-788.

Zardo, K. M., Santos, D. R., Babicsak, V. R., Belotta, A. F., Oliveira, H. S., Estanislau, C. A., ... \& Brandão, C. V.S. 2012. Aspecto ultrassonográfico da dioctofimose renal canina. Veterinária e Zootecnia, 19, 57-60.

Zolhavarieh, S. M., Norian, A. \& Yavari, M. 2016. Dioctophyma renale (Goeze, 1782) Infection in a Domestic Dog from Hamedan, Western Iran. Iranian Journal of Parasitology, 11, 131135.

Recebido: 10 Agosto., 2018

Aprovado: 30 Agosto.,. 2018

Publicado: 11 Setembro 2018

Licenciamento: Este artigo é publicado na modalidade Acesso Aberto sob a licença Creative Commons Atribuição 4.0 (CC-BY 4.0), a qual permite uso irrestrito, distribuição, reprodução em qualquer meio, desde que o autor e a fonte sejam devidamente creditados. 